BULL. AUSTRAL. MATH. SOC.

MOS 2025, 2040, (2008)

VOL. $2(1970), 347-357$.

\title{
The formation generated by a finite group
}

\section{R. M. Bryant, R. A. Bryce and B. Hartley}

\begin{abstract}
We prove here that the (saturated) formation generated by a finite soluble group has only finitely many (saturated) subformations. This answers a question asked by Professor $W$. Gaschütz. Some partial results are also given in the case of a formation generated by an arbitrary finite group.
\end{abstract}

At the Ninti Summer Research Institute of the Australian Mathematical Society, held in Canberra in 1969, Professor W. Gaschütz asked the following questions [3,7.22]: "Does the (saturated) formation generated by a finite soluble group contain only finitely many (saturated) subformations?" We show in $\$ 1$ that the answer to each of these questions is "Yes". The proof relies on that of the Oates-Powell theorem - 52.11 in Hanna Neumann [5] - which answers the corresponding question for the variety generated by a finite group. We have unsuccessfully considered the same question for the formation generated by a finite insoluble group. The best we can do in general is described in $\S 2$, where we show that among the subformations generated by quotients of direct powers of the generating group only finitely many are distinct. Incidentally, a corollary of Lemna 1.5 below - Corollary 1.6 - is the result of Peter $M$. Neumann [6] that a formation consisting of nilpotent groups is subgroup closed.

We refer the reader to the paper [1] of Carter, Fischer and Hawkes for notation and definitions relating to formations. In addition we remark that if $\underline{\underline{X}}$ is a class of groups then the formation generated by $\underline{\underline{X}}$

Received 18 February 1970. 
is just $Q R_{0} \underline{X}$ : this follows since $R_{0} Q \underline{\underline{X}} \subseteq Q R_{0} \mathrm{X}$ - if $X$ is a subdirect product of groups $X_{1} / N_{1}, \ldots, X_{p} / N_{p}$, where each $X_{i} \in \underline{\underline{X}}$, then clearly $X$ is a homomorphic image of a subdirect product of $X_{1}, \ldots, X_{r}$ under the natural homomorphism of $X_{1} \times \ldots \times x_{r}$ onto $X_{1} / N_{1} \times \ldots \times X_{r} / N_{r}$.

\section{The soluble case}

We denote by form $(G)$ the formation generated by the group $G$. A finite group $G$ will be called formation critical if the formation generated by those proper factors of $G$ which lie in form $(G)$ does not contain $G$ (cf. 51.31 in [5]). Every formation is generated by its formation critical groups (cf. 51.41 in [5]). We remind the reader that a Cross variety is a variety generated by a single finite group. We shall prove

LEMMA 1.1. A soluble Cross variety contains only finitely many formation aritical groups.

The remarks preceding Lemma 1.1 then yield

THEOREM 1.2. The formation which consists of the finite groups of a soluble cross variety has only finitely many subformations.

Since the formation generated by a group is contained in the variety it generates this is enough to answer one of the questions of Gaschütz cited in the introduction. The other question can be answered easily using Theorem 1.2.

COROLLARY 1.3. The saturated formation generated by a finite soluble group contains only finitely many saturated subformations.

Proof. We appeal to the well-known theorems of Gaschütz and Lubeseder (B. Huppert [4, VI.7.5 and VI.7.25]) that a formation of finite soluble groups is saturated if and only if it can be locally defined.

Let $F$ be the saturated formation generated by a finite soluble group $G$ and let $\pi$ be the set of primes dividing $|G|$. For $p \in \pi$, define $F(p)=$ form $\left(G / 0_{p^{\prime}} p^{(G))}\right.$, and define $F(p)=\emptyset$ otherwise: then it follows by the theorems just mentioned that $F$ is the (saturated) formation defined locally by the $F(p)$. (Here, as usual, ${ }^{0} p^{\prime} p^{(G)}$ 
denotes the largest $p$-nilpotent normal subgroup of $G$. )

Let $G$ be any saturated subformation of $F$, defined locally by formations $G(p)$. Then $G(p)=\emptyset$ for $p \notin \pi$. Furthermore, by replacing $G(p)$ by $G(p) \cap F(p)$ if necessary, we may suppose that $G(p) \subseteq F(p)$, $p \in \pi$. Now Theorem 1.2 ensures that each $F(p)$ has only finitely many subformations, and $\pi$ is finite, so there are only finitely many possibilities for $G$.

The proof of Lemma 1.1 depends on the next two lemmas.

LEMMA 1.4. If $Y$ is a subgroup of a nilpotent group $X$ whose class is smaller than that of $X$ then the class of the normal closure $Y$ of $Y$ in $X$ is also smaller than the class of $X$.

Proof. It will be enough to show (we do it by induction on $d$ ) that

$$
\gamma_{d}\left(Y^{X}\right) \leq \gamma_{d+1}(X) \gamma_{d}(Y), d \in\{1,2, \ldots\} \text {. }
$$

For $d=1$ this is certainly the case since, for $x \in X, y \in Y$, $y^{x}=\left[x, y^{-1}\right] y ;$ hence

$$
\gamma_{1}\left(Y^{X}\right)=Y^{X} \leq \gamma_{2}(X) Y=\gamma_{2}(X) \gamma_{1}(Y) \text {. }
$$

Assume that (*) has been proved for some $d \geq 1$. Then

$$
\begin{aligned}
\gamma_{d+1}\left(y^{X}\right) & =\left[\gamma_{d}\left(Y^{X}\right), Y^{X}\right] \leq\left[\gamma_{d+1}(X) \gamma_{d}(Y), Y^{X}\right] \\
& =\left[\gamma_{d+1}(X), Y^{X}\right]\left[\gamma_{d}(Y), Y^{X}\right] \leq \gamma_{d+2}(X)\left[\gamma_{d}(Y), \gamma_{2}(X) Y\right] \\
& =\gamma_{d+2}(X)\left[\gamma_{d}(Y), Y\right]\left[\gamma_{d}(Y), \gamma_{2}(X)\right] \leq \gamma_{d+2}(X) \gamma_{d+1}(Y)
\end{aligned}
$$

as required.

LEMMA 1.5. Every supplement of the Fitting subgroup of a finite group $G$ is in the formation generated by $G$.

Proof. Let $S$ be a subgroup of $G$ and $T$ a nilpotent normal subgroup of $G$ such that $G=S T$. We show, by induction on the class of $T$, that $S \in \operatorname{form}(G)$.

Consider the following subgroups of $G \times G \times G$ :

$$
K=\{(s, s, s): s \in S\},
$$




$$
D_{1}=\{(t, t, 1): t \in T\} \text { and } D_{2}=\{(1, t, t): t \in T\} \text {. }
$$

Let $H$ be the subgroup they generate: then $H$, being subdirect, is in form $(G)$. Note that $D_{1}$ and $D_{2}$ are each normalized by $K$.

If $T$ is abelian then $D_{1}$ and $D_{2}$ centralize each other. Also $D_{1} D_{2} \cap K=I$ because an arbitrary element of $D_{1} D_{2}$ may be written in the form $(t, t u, u)$ for some $t, u \in T$ and it is in $K$ only if $t=t u=u$, that is only if $t=u=1$. Consequently, when $T$ is abelian,

$$
S \cong K \cong H / D_{1} D_{2} \in \operatorname{form}(G)
$$

Suppose now that the class $c$ of $T$ is greater than 1 . Put $M=Z\left(D_{1}\right) Z\left(D_{2}\right)$ and note that, as in the last paragraph, $M \cap K=1$. Also $M$ is normal in $H$. Since

$$
\left[\gamma_{c-1}\left(D_{1}\right), D_{2}\right]=\left\{(1, w, I): w \in \gamma_{c}(T)\right\} \ddagger M,
$$

the groups $D_{1} M / M$ and $D_{2} M / M$ generate a subgroup $C$ of $H / M$ of class $c$ exactly. However $D_{2} M / M$ has class $c-1$ and so its normal closure $B$ in $C$ also has class $C-1$, by Lemma 1.4. But $B$ is normal in $H / M$ and so, by induction, $K D_{1} M / M \in$ form $(H / M)$. Finally $D_{1} M / M$ is normal in $K D_{1} M / M$ and of class $c-1$, so, again by induction, $K M / M \in \operatorname{form}\left(K D_{1} M / M\right)$. Therefore

$$
S \cong K \cong K M / M \in \text { form }(G)
$$

The result of Peter $M$. Neumann referred to in the introduction follows immediately from Lemma 1.5 .

COROLLARY 1.6. A formation consisting of nilpotent groups is subgroup closed.

We shall also require the following version of 51.37 of [5]: this consists simply of a rather precise statement of what emerges in the course of the proof of 51.37, and we refer the reader to [5] for details.

LEMMA 1.7. Let $A$ be a finite group which is generated by the subgroup $L$ together with normal subgroups $M_{1}, M_{2}, \ldots, M_{\mathcal{S}}$. Let $\underline{\underline{\mathrm{x}}}$ be the set of subgroups of $A$ which can be generated by $L$ together with some proper subset of $\left\{M_{1}, M_{2}, \ldots, H_{s}\right\}$. 
Suppose that

$$
\left[M_{\pi(1)}, M_{\pi(2)}, \ldots, M_{\pi(s)}\right]=1
$$

for every permutation $\pi$ of $\{1,2, \ldots, s\}$. Then $A$ is in $Q R$ of .

Proof of Lemma 1.1. We shall follow closely the proof of 52.23 of [5]. In particular Lemma 1.7 will be used in place of 51.37 of [5].

Let $\underline{\underline{v}}$ be a Cross variety. Then the results of Chapter 5 of [5] show that there are bounds on

(a) the class of a nilpotent group in $\underline{\underline{V}}$,

(b) the order of a chief factor of a group in $\underline{\underline{V}}$, and

(c) the order of a finite group in $\underline{\underline{V}}$ on a given number of generators.

Suppose that $\underline{V}$ is soluble and that $A$ is a formation critical group in $\underline{\underline{\mathrm{V}}}$. Let $F$ and $\Phi$ be, respectively, the Fitting and Frattini subgroups of $A$. By the results of $W$. Gaschütz [2] we know that $F / \Phi$ is the direct product of minimal normal subgroups $M_{1} / \Phi, M_{2} / \Phi, \ldots, M_{s} / \Phi$ of $A / \Phi$ and that $F / \Phi$ has a complement $L / \Phi$ in $A / \Phi$. Also $F / \Phi$ is the Fitting subgroup of $A / \Phi$ and so $C_{A}(F / \Phi)=F$ by W.R. Scott $[7,7.4 .7]$.

Suppose that $s$ exceeds the bound given by (a) above. Then the groups $L$ and $M_{1}, M_{2}, \ldots, M_{s}$ satisfy the conditions of Lemma 1.7; and furthermore the groups in the set $X$ of Lemma 1.7 all lie in form $(A)$ by Lema 1.5. Thus we deduce that $\operatorname{form}(A)$ is generated by $\underline{\underline{X}}$. But $A \notin \underline{\underline{\mathrm{X}}}$, and so this contradicts the definition of $A$.

Consequently (a) and (b) give a bound on the order of $F / \Phi$. Therefore we also have that $|A: F|=\left|A: C_{A}(F / \Phi)\right|$ is bounded. Thus we obtain a bound on $|A: \Phi|$ and hence a bound for the number of generators needed for $A$. Finally (c) gives a bound on the order of $A$ and completes the proof of Lemma 1.1 .

To conclude this section we record the following result which is presumably well-known (cf. VI.7.2l of [4]).

LEMMA 1.8. Let $N$ be a normal subgroup of a group $G$ and $M a$ 
normal subgroup of $G$ which centralizes $N$. Then the group $G^{*}$ obtained by split extending $N$ by $G / M$ with its natural action on $N$ is in the formation generated by $G$.

Proof. Let $G_{1}=\{(g, g): g \in G\}$ and $N_{1}=\{(n, 1): n \in N\}$ be subgroups of $G \times G$; and note that $G_{1}$ normalizes $N_{1}$. Put $M_{1}=\{(m, m): m \in M\}$, so that $M_{1}$ is normalized by $G_{1}$ and centralized by $N_{1}$. Then, clearly,

$$
G^{*} \cong G_{1} N_{1} / M_{1} \in \text { form }(G) .
$$

\section{Direct powers and formations}

Throughout this section $G$ will denote a given finite group and $g \rightarrow g^{*}, g \rightarrow g_{i}$ and $g \rightarrow g_{i}^{*}$ will denote fixed isomorphisms of $G$ onto groups $G^{*}, G_{i}$ and $G_{i}^{*}$, respectively. We shall employ the natural consequences of this notation - thus if $K$ is a subgroup of $G$ then $K_{i}$ will denote the subgroup of $G_{i}$ corresponding to $K$, and so on.

Let $U \leq V$ be normal subgroups of $G$ with $[V, G] \leq U$ and let $D(U, V)$ be the subgroup of $G \times G^{*}$ consisting of all elements $v w^{*}$ with $v, w \in V$ and $v \equiv w$ modulo $U$. Then $D(U, V) \triangleleft G \times G^{*}$. By a central factor square of $G$ we shall mean a group isomorphic to a group $G \times G^{*} / D(U, V)$. Since any two isomorphisms $G \rightarrow G^{*}$ differ by an automorphism of $G$, such a group is determined up to isomorphism by $U$ and $V$ and is independent of the particular isomorphism $g \rightarrow g^{*}$ selected.

The main result of this section is the following.

THEOREM 2.1. Let $H$ be a homomorphic image of a direct power of $G$. Then the formation generated by $H$ can be generated by a central factor square $G \times G^{*} / D(U, V)$ of $G$ such that $U$ and $V$ are characteristic in $G$ and the cutomorphism group of $G / U$ acts trivially on $V / U$.

Proof. The proof will be conducted by induction on $|G|$. If $|G|=1$ the theorem clearly holds and so we may assume that $|G|>1$ and that the theorem holds for all groups of order smaller than $|G|$. 
Suppose then that

$$
H=G_{1}^{*} \times G_{2}^{*} \times \ldots \times G_{l}^{*} / X
$$

where $Z^{*} \geq 1$ and $X \triangleleft D=G_{1}^{*} \times G_{2}^{*} \times \ldots \times G_{l}^{*}$. Let $\bar{X}=\cap X^{\alpha}$, the intersection being taken over all $\alpha \in$ Aut $D$. Then clearly $D / \bar{X}$ and $H$ generate the same formation and so we may assume that $X$ is characteristic in $D$. We now distinguish two cases.

Case $1: X$ contains $Y_{1}^{*} \times \ldots \times Y_{l}^{*}$ for some $l \neq Y \leq G$. Then $X \cap G_{1}^{*}$ is a non-trivial characteristic subgroup $T_{1}^{*}$ of $G_{1}^{*}$ and, since the symmetric group $S_{l}$ acts naturally on $D, X$ contains $T_{1}^{*} \times \ldots \times T_{l}^{*}$. But then $H$ is a homomorphic image of a direct power of $G / T$ and so, by our inductive hypothesis, form $(H)$ can be generated by a central factor square of $G / T$ constructed with respect to characteristic subgroups $U / T$ and $V / T$ of $G / T$, with the properties described. The central factor square is isomorphic to $G \times G^{*} / D(U, V)$ : furthermore $U$ and $V$ are characteristic in $G$ and Aut $G / U$ acts trivially on $V / U$.

Case 2: $X$ contains no subgroup of the form $Y_{1}^{*} \times \ldots \times Y_{l}^{*}$ with $I \neq Y \leq G$. Let

$$
E=G_{1} \times G_{2} \times \ldots \times G_{Z+1}
$$

and let $L$ be the intersection of the kernels of the homomorphisms of $E$ onto $H$. If $\phi$ is any such homomorphism and $\alpha \in$ Aut $E$ then $\alpha \phi$ is another homomorphism of $E$ onto $H$ with kernel $(\operatorname{ker} \phi)^{\alpha^{-1}}:$ thus $L$ is characteristic in $E$.

We shall show that $L$ contains no element of the form

$$
t=z_{i} w_{j} x
$$

where $i \neq j, z$ and $w$ are distinct elements of $G$, and $x \in \prod_{k \neq i, j} G_{k}$. In fact suppose that $L$ does contain such an element. Then, since the symmetric group $S_{l+1}$ acts on $E$, we find that $L$ also contains $u=w_{i} z_{j}$. Hence $L$ contains $t u^{-1}=y_{i} y_{j}^{-1}$ where 
$y=z w^{-1} \neq 1$, and by replacing $y$ by a suitable power we may assume that $y$ has prime order $p$. By applying further elements of $S_{\eta+1}$ we find that $L$ contains every element $y_{k} y_{m}^{-1}$ with $k \neq m$ and so if $y=\langle y\rangle$ and $Z=Y_{1} \times \ldots \times y_{\eta+1}$ we have

$$
|z: Z \cap L| \leq p \text {. }
$$

However let $\psi$ be the homomorphism of $E$ onto $H$ determined by the conditions $g_{i} \rightarrow g_{i}^{*} X(i \neq z+1)$ and $g_{\eta+1} \rightarrow 1$. Then by the assumptions of Case 2 we have $y_{j} \ddagger \operatorname{ker} \psi$ for some $j$ with $1 \leq j \leq l$. On the other hand there is a homomorphism $\chi$ of $E$ onto $H$ whose kernel contains $y_{j}$, and even the whole of $G_{j}$, but fails to contain some other $y_{k}$. Therefore $Z \cap$ ker $\psi$ and $Z \cap$ kerX are distinct proper subgroups of $Z$ and so

$$
|z: z \cap \operatorname{ker} \psi \cap \operatorname{ker} x| \geq p^{2} \text {. }
$$

This contradicts (2) and shows that $L$ contains no element of the form (1).

The set of all elements $g \in G$ such that $\prod_{i=1}^{z+1} g_{i} \in L$ is a subgroup $V$ of $G$. Now if $\alpha$ is an automorphism of $G$ and $h$ an element of $V$ then $\left(h^{\alpha}\right)_{1} \prod_{i=2}^{l+1} h_{i}$ is in $L$, and consequently $h^{\alpha}=h$ by what we have just proved. Hence Aut $G$ fixes every element of $V$ and in particular $V$ is characteristic in $G$.

Now clearly $H$ and $E / L$ generate the same formation. However let

$$
k_{i j}=\prod_{k \neq i, j} G_{k}\left\langle v_{i} v_{j}: v \in V\right\rangle(1 \leq i, j \leq \imath+1, i \neq j) \text {. }
$$

Then $E / K_{i j} \cong G \times G^{*} / D(I, V)$ and $n K_{i j}=L$. Consequently $E / L$ generates the same formation as $G \times G^{*} / D(1, V)$, and the proof is complete.

COROLLARY 2.2. Only finitely many formations can be generated by subsets of $Q D_{0}(G)$. 
(Here $D_{0}(G)$ denotes the class of isomorphic copies of direct powers of $G$. )

Proof. Let $F$ be a formation generated by groups $H_{1}, H_{2}, \ldots$ belonging to $Q D_{0}(G)$. By Theorem 2.1 the formation generated by $H_{i}$ can be generated by one of the groups $G \times G^{*} / D(U, V)$ and so each $H_{i}$ can be replaced by one of these groups. Thus there are only finitely many possibilities for $F$.

It may be worth remarking that the formations generated by the groups $G \times G^{*} / D(U, V)$ are not necessarily distinct as can be seen by taking $G$ to be cyclic of order two.

\section{The formation generated by $\operatorname{SL}(2,5)$}

As an application of Theorem 2.1 we shall briefly indicate how the lattice of subformations of the formation $F$ generated by $G=S L(2,5)$ may be determined. It will be clear that the method is of more general application, but in the interests of brevity we shall refrain from stating a cumbersome general result. Much of the detail will be left to the reader.

In the sequel $G_{1}, G_{2}, \ldots$ will denote isomorphic copies of $G$ and $z_{i}$ will be the centre of $G_{i}$, with $z_{i}=\left\langle z_{i}\right\rangle$.

Firstly we remark that $G$ has the following property.

(3) If $M$ is a finite group with a central subgroup $W$ such that $M / W \cong G$ then $M$ splits over $W$.

Proof. By [4, V.25.4] the Schur multiplicator of $G$ is trivial. Let $\phi$ be an epimorphism of a free group $F$ of finite rank onto $M$ and let $R=\phi^{-1}(W)$. Then $F / R \cong G$, and, since the Schur multiplicator of $G$ is isomorphic to $R \cap F^{\prime} /[F, R] \quad$ (V.23.5 of [4]), we have $R \cap F^{\prime} \leq[F, R]$. Therefore $M^{\prime} \cap W=1$ and $M=M^{\prime} \times W$.

It is clear that any group $L \in F$ has a central subgroup $W$ of exponent 2 such that $L / W$ belongs to the formation generated by the alternating group $A_{5}$. Furthermore the latter formation consists of all finite direct powers of $A_{5}$; and so $L / W$ is perfect. Therefore 
$L=L^{\prime} W$. It follows that $L^{\prime}=L^{\prime \prime}$ and that if $U$ is a complement for $L^{\prime} \cap W$ in $W$ then $L=L^{\prime} \times U$. Notice that, by Lemma 1.8, every central subgroup of $G$ belongs to $F$, and so $F$ contains the cyclic group $C_{2}$ of order 2 .

Now we have already pointed out that form $(G)=Q R_{0}(G)$. We shall show that any group $H \in R_{0}(G)$ is a direct product of copies of $G$ and of $C_{2}$. By the remarks made in the preceding paragraph it is enough to consider the case where $H$ is perfect and is subdirectly contained in $G_{1} \times \ldots \times G_{n}$ for some $n$. Our assertion follows by induction on $n$ unless ' $H \cap G_{1}=Z_{1}$, and we shall now exclude this possibility.

Suppose then that $H \cap G_{1}=Z_{1}$. Induction shows that $H=A_{1} A_{2} \ldots A_{k}$ where the $A_{i}$ are normal subgroups of $H$ containing $z_{1}$ such that $A_{i} / Z_{1} \cong G$ and $A_{i} \cap A_{j}=Z_{1}$ if $i \neq j$. By (3) we have $A_{i}=z_{1} \times B_{i}$ with $B_{i} \cong G . B_{i}$ is characteristic in $A_{i}$ and so normal in $H$, and it follows easily that $H=2_{1} \times B_{1} \times \ldots \times B_{k}$. As $H=H^{\prime}$ we have a contradiction.

It now follows that any group $L \in F$ has the form

$$
\left(G_{1} \times \ldots \times G_{2} / X\right) \times C
$$

where $Z \geq 0, X \leq Z_{1} \times \ldots \times Z_{Z}$ and $C$ is elementary abelian of exponent 2 . If $x=z_{1} \times \ldots \times z_{\eta}$ it is clear that the formation generated by $L$ can be generated by one of the groups $1, C_{2}, A_{5}$ and $A_{5} \times C_{2}$. Otherwise, by Theorem 2.1, the formation generated by $L$ can be generated either by $G$ or by the central square $S$ of $G$.

Finally we show that the formations $F$ and $F^{*}$ generated by $G$ and $S$ are distinct. Otherwise $G \in F^{*}$ and $G$ would be a homomorphic image of a group $G_{1} \times \ldots \times G_{2} / X$ with $X \leq Z_{1} \times \ldots \times Z_{\eta}$ and $X$ an intersection of kernels of homomorphisms of $G_{1} \times \ldots \times G_{\tau}$ onto $S$. It is easy to see that any such kernel contains the element $\bar{z}=z_{1} \ldots z_{l}$. Therefore $G$ would be a homomorphic image of the group $G_{1} \times \ldots \times G_{l} /\langle\bar{z}\rangle$. However this is not the case and so $G \notin F^{*}$. 
Thus $F$ has precisely six subformations. They are generated respectively by the groups $I, C_{2}, A_{5}, A_{5} \times C_{2}, S$, and $G$ itself.

\section{References}

[1] Roger Carter, Bernd Fischer and Trevor Hawkes, "Extreme classes of finite soluble groups", J. Azgebra 9 (1968), 285-313.

[2] Wolfgang Gaschütz, "Über die $\Phi$-Untergruppe endlicher Gruppen", Math. 2. 58 (1953), 160-170.

[3] Wolfgang Gaschütz, Selected topics in the theory of soluble groups (Lectures given at the Ninth Summer Research Institute of the Australian Mathematical Society in Canberra, 1969. Notes by J. Looker).

[4] Bertram Huppert, EndZiche Gruppen I (Die Grundlehren der mathematischen Wissenschaften, Band 134, Springer-Verlag, Berlin, Heidelberg, New York, 1967).

[5] Hanna Neumann, Varieties of groups (Ergebnisse der Mathematik und ihrer Grenzgebiete, Band 37, Springer-Verlag, Berlin, Heidelberg, New York, 1967).

[6] Peter M. Neumann, "A note on formations of finite nilpotent groups", Bulz. London Math. Soc. 2 (1970), 91.

[7] W.R. Scott, Group theory (Prentice-Hall, Englewood Cliffs, New Jersey, 1964).

The Australian National University, Canberra, ACT. 